
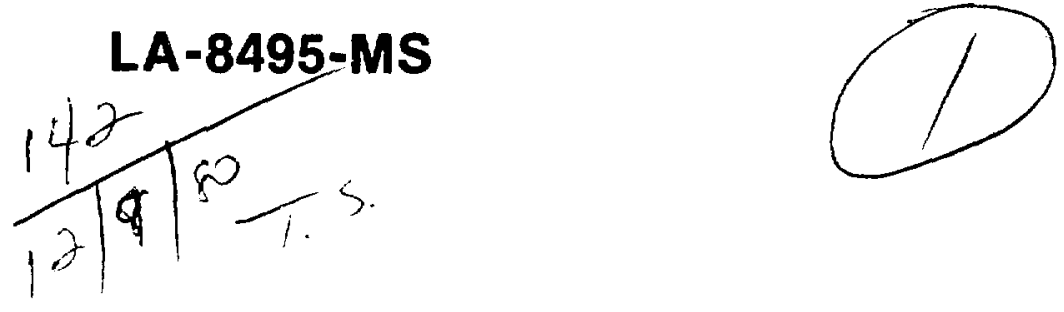

Dr. 2107

\title{
Influence of a Vacuum Region on the Stability of a High-Beta Screw Pinch
}

\section{MASTER}

烍

$\frac{7}{\infty}$

文 


\section{Influence of a Vacuum Region on the Stability of a High-Beta Screw Pinch}

Thomas E. Cayton

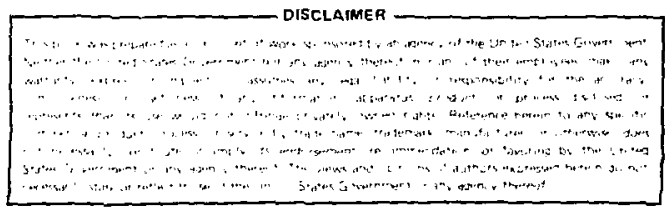


INFLUENCE OF A VACUUM REgION ON THE STABILITY OF A HIGH-BETA SCREW PINCH

by

Thomas E. Cayton

\begin{abstract}
To ascertain the influence of a vacuum region on the stablilty of a high-B screw pinch, the stability properties of two confinement configurations are compared. Both configurations involve diffuse equilibrium profiles and a rigid, perfectly conducting cylindrical shell. In the first problem, perfectly conducting plasma extends to the rigid conducting wa11; the plasma is extremely tenuous in the oucer region of the pinch, however. In the second case, profiles Identical to those of the first problem are chosen for the central portion of the pinch, but the outer tenuous plasma 18 replaced by a perfectly insulating vacuum region. The two configurations are fourd to be unstable for the same range of external parameter values; different modes are unstable in the two cases, however. Thus, the presence of a vacuum region does not affect the stability boundary of the pinch, but it does affect the nature of the ungtable modes.
\end{abstract}

\title{
I. INTRODUCTION
}

In Ideal magnetohydrodynamic (MDi) stabllity analyses of high- $\beta$ pinch configurations, an uncertainty concerning the outer portion of the pinch 18 frequently encountered. In many instances, 3xperimental measurements suggest equilibrium profiles in which current density, pressure, and asse density in the outer region are negligible compared with their values in the central portion of the pinch. Therefore, in the outer region, the magnetic field closely approximates a vacuum field. The uncertainty is this. Does the plasma really extend to the vacuum vessel, or does a vacuum region separate the plasna column and the wall? The boundary conditions appropriate for a Iinear stablity analys18 are different for the two configurations; therefore, the eigenvalues that result may also differ. The objective of this report is 
to shed some light on the Influence which the outer portion of the pinch exerts on MHD stability.

To ascertain the influence of a vacuum region on the stability of a high-B screw pinch, we compare the stabllity properties of two configurations. Both configurations involve diffuse equilibrium profiles and a rigid, perfectly conducting cylindrical shell. In the first problem, perfectly conducting plasma extends to the rigid conducting wall; the plasma is extremely tenuous in the outer region of the pinch, however. In the second problem, profiles identical to those of the first problem are chosen for the central portion of the pinch, but the outer tenuous plasma is replaced by a perfectly insulating vacuum region. On one hand, the nature of the equilibrium magnetic fleld in the outer region, together with the very small mass density suggest that there is little distinction between the two configurations. But on the other hand, the electrical conductivity is uniform throughout the domain of inter st in one case, while in the other case an Interface between perfectly conducting plasma and perfectly insulating vacuum divides the domain. It is well known that certain perturbations (surface waves) propagate along such surfaces of discontinuity. Sirce these perturbations may be unstable, and because surface waves have no , zounțerpirt in continuous media, a real distinction may exist between the two configurations. We shall find that the presence of a vacuum region does not affect the stability boundary of the pinch. It does affect the nature of the unstable modes, and their growth rates, however.

The report 18 organized as follows. The stability problems for the two configurations are formulated in Sec. II. Numerical results are presented in Sec. III. Results are summarized and conclusions are presented in Sec. IV.

\section{TWO STABILITY PROBLEMS}

We use the Iinearized equations of Ideal magnetohydrodynamics in standard cylinirical polar coordinates to examine the stability of diffuse screw pinch equilibria. The equilibrium density, $\rho$, pressure, $p$, and the magnetic field components, $B_{\theta}$ and $B_{z}$, are functions only of the radial coordinate, $r$ and they satisfy the equations of ideal magnetohydrostatics, 


$$
\left[p+\frac{1}{2}\left(B_{\theta}^{2}+B_{z}^{2}\right)\right]^{-}+\frac{B_{\theta}^{2}}{r}=0,
$$

Where a prime denotes diffentiation with respect to $r$. We use a system of untts whose characteristic length, mass, and time are defined in terms of the following physical quantitles: 1) the equivalent sharp-boundary radius of the pinch, a; 2) the mass density measured on axis, $\rho_{0} ; 3$ ) The magnetic field measured for from the plasma, $B_{z \infty}$.

Numerical results presented in Sec. TII are obtained using the rigid-rotor profiles of Ref. 1,

$$
\begin{aligned}
& \rho(r)=\rho_{0} \frac{\operatorname{sech}^{2}\left(r^{2} / r_{0}^{2}+\alpha\right)}{\operatorname{sech}^{2} \alpha}, \\
& B_{z}(r)=B_{2 \infty} \tanh \left(r^{2} / r_{0}^{2}+\alpha\right), \\
& B_{\theta}(r)=\mu B_{2 \infty}\left(\frac{r_{0}}{r}\right) \frac{\tanh \left(r^{2} / r_{0}+\alpha\right)-\tanh \alpha}{1-\tanh \alpha},
\end{aligned}
$$

where the parameter $\alpha$ is related to the plasma $\beta$ by $B=\operatorname{sech}^{2} \alpha$, and $r_{0}=[1+$ $\left.(1-B)^{1 / 2}\right]^{1 / 2}$ a.

Equations (1)-(4) specify the equilibria in terms of two parameters, $A$ and $\mu$. We shall consider two specific equilibria. In the first example, the plasma is assumed to extend to a rigid perfectly c. iducting cylindrical shell located at $r=I_{w}$; we shall designate this as the $P-W$ system. In the second case, the plasma region consists of a cylinder of radius $r_{p}$ which is enclosed by a perfectly conducting shell of radius $r_{w}>r_{p}$; a vacuum region exists between the cylindrical surfaces $r=r_{p}$ and $r=r_{w}$. We shall designate the second configuration as $\mathrm{P}-\mathrm{V}-\mathrm{W}$ system. The two configurations have identical equilibrium profiles for $r<r_{p} \cdot r_{p}$ is chosen sufficiently large so that $\rho\left(r=r_{p}\right) \ll \rho(r=0)$ and also so that the wagnetic field closely approximates a vacuu field. Surface currents are permitted to flow along the plasma-vacuum interface of the P-V-W system in order that the two configurations exhibit the same values for the magnetic field components at 
the conducting wall, $r=r_{w}$. Thus, the two configurations carry the same axial current. The essential difference between them is that the tenuous outer region in the $P-W$ system is replaced by a vacuum region in the $P-V-W$ system. This difference 18 manifest in the profiles of the electrical conductivity. The electrical conductivity is uniform throughout the domain of Interest, $\left(0, r_{w}\right)$, for the $P-W$ system; an interface between perfectly conducting plasma and perfectly Insulating vacuum divides the domain of the P-V-W system.

The displacement formulation of Iinearized MHD is used for the stability analyses. Because of the symmetries of the equilibria, perturbation quantities are assumed to be of the form,

$$
f(r, \theta, z, t)=f(r) \exp [1(m \theta+k z-\omega t)]
$$

where $m, k$, and $w$ are parameters. These parameters must be chosen so that the displacement, $\xi_{z}=\xi_{\mathbf{r}} \hat{\underline{r}}+\xi_{\theta} \hat{\theta}+\xi_{z} \hat{z}$, satisfies the equation of motion,

$$
-\rho \omega^{2} E+\nabla P=\underline{T}
$$

and approprlate boundary conditions. The relationship between $w$ and the other parameters is expressed by the other dispersion differential equation whicis is derived from Eq. (5). In Eq. (5), $P$ is the total perturbed pressure and $\underline{T}$ is the tension vector, both of which may be expressed in terms of $\xi_{r}, \xi_{\theta}$, and $\xi_{z}$, and equflibrium quantities. When algebraic quantities are eliminated, Eq. (5) becomes

$$
\begin{aligned}
& \left\{\frac{\left(\rho \omega^{2}-F^{2}\right)\left[\rho \omega^{2}\left(\gamma p+B^{2}\right)-\gamma p F^{2}\right]}{r \Delta}\left(r \xi_{r}\right)^{-}\right\}^{-}+\left(\rho \omega^{2}-F^{2}\right) \xi_{r} \\
& -2 B_{\theta}\left(\frac{B_{\theta}}{r}\right)-\xi_{r}-\frac{4 k^{2} B_{\theta}^{2}}{r^{2} \Delta}\left(\rho \omega^{2} B^{2}-\gamma p F^{2}\right) \xi_{r} \\
& -r\left(\frac{2 k B_{\theta}\left(k B_{\theta}-\frac{m}{r} B_{z}\right)\left[\rho \omega^{2}\left(\gamma p+B^{2}\right)-\gamma p F^{2}\right]}{r^{2} \Delta}-\xi_{r}=0,\right.
\end{aligned}
$$


where $Y$ is the ratio of specific heats,

$$
\begin{aligned}
& B^{2} \equiv B_{\theta}^{2}+B_{z}^{2}, \\
& F \equiv k B_{z}+\frac{m}{r} B_{\theta}, \\
& \Delta \equiv\left(\rho \omega^{2}\right)^{2}-\left(k^{2}+\frac{m^{2}}{r^{2}}\right)\left[\rho \omega^{2}\left(\gamma p+B^{2}\right)-\gamma p F^{2}\right] .
\end{aligned}
$$

\section{A. P-W Stability}

Equation (6) applies to the entire domain. Appropriate boundary conditions are

$$
\xi_{r}(r=0)=\text { finite, }
$$

and

$$
\xi_{r}\left(r=r_{w}\right)=0
$$

Equations (6), (10), and (11) determine $\omega$ and $\xi_{r}(r)$ for given $m, k$, and equilibrium profiles $p(r), p(r), B_{\theta}(r)$, and $B_{z}(r)$.

\section{B. P-V-W Stability}

Equation (6) applles only to the plasma region. In the vacuum region, the source-free Maxwell equations determine the electromagnetic field. The solutions in the two regions are matched at the plasma-vacuum interface. This Interface is displaced from its equilibrium position, $r=r_{p}$, and its shape is distorted by the perturbetion. The linearized equation of the perturbed Interface 18

$$
r=r_{p}+\xi_{r}\left(r_{p}\right) \exp [1(m \theta+1 k z-1 t)]
$$


The unit normal, $\hat{n}$, to the surface of the perfectly conducting plasma is

$$
\hat{\underline{n}}=\hat{\underline{r}}-1\left(\frac{m}{r_{p}} \hat{\theta}+k \hat{z}\right) \xi_{r}\left(r_{p}\right) \exp [1(m \theta+k z-\omega t)] .
$$

In the vacuum region bounded by the perturbed piasme-vacuum interface and the rigld, perfectly conducting wall at $c=r_{w}$, the perturbed magnetic field has the following components.

$$
\begin{aligned}
& \delta B_{r}^{v}=1\left(\frac{m}{r_{p}} B_{\theta}^{v}+k B_{z}^{v}\right) \xi_{r}\left(r_{p}\right) \frac{\left[K_{m}^{\prime}(|k| r)-I_{m}^{\prime}(|k| r) \frac{K_{m}^{\prime}\left(|k| r_{w}\right)}{\Gamma_{m}^{\prime}\left(|k| r_{w}\right)}\right]}{\left[K_{m}^{\prime}\left(|k| r_{p}\right)-I_{m}^{\prime}\left(|k| r_{p}\right) \frac{K_{m}^{\prime}\left(|k| r_{w}\right)}{I_{m}^{\prime}\left(|k| r_{w}\right)}\right]}, \\
& \delta B_{\theta}^{V}=-\frac{m}{|k| r}\left(\frac{m}{r_{p}} B_{\theta}^{v}+k B_{z}^{v}\right) \xi_{r}\left(r_{p}\right) \frac{\left[K_{m}(|k| r)-I_{m}(|k| r) \frac{K_{m}^{\prime}\left(|k| r_{w}\right)}{I_{m}^{\prime}\left(|k| r_{w}\right)}\right]}{\left[K_{m}^{\prime}\left(|k| r_{p}\right)-I_{m}^{\prime}\left(|k| r_{F}^{\prime} \frac{K_{m}^{\prime}\left(|k| r_{w}\right)}{I_{m}^{\prime}\left(|k| r_{w}\right)}\right]\right.}, \\
& \delta B_{z}^{v}=-\frac{k}{|k|}\left(\frac{m}{r_{p}} B_{\theta}^{v}+k B_{z}^{v}\right) \xi_{r}\left(r_{p}\right) \frac{\left[K_{m}(|k| r)-I_{m}(|k| r) \frac{K_{m}^{\prime}\left(|k| r_{w}\right)}{I_{m}^{\prime}\left(|k| r_{w}\right)}\right]}{\left[K_{m}^{\prime}\left(|k| r_{p}\right)-I_{m}^{\prime}\left(|k| r_{p}\right) \frac{K_{m}^{\prime}\left(|k| r_{w}\right)}{I_{m}^{\prime}\left(|k| r_{w}\right)}\right]},
\end{aligned}
$$

where $K_{m}$ and $I_{m}$ are modifled Bessel functions of order $m$, primes designate differentiation of these functions with respect to thetr argunent, and $B_{\theta}^{\gamma}$ and $B_{z}$ are the $\theta$ and $z$ components of the equilibrium magnetic field in the vacuun region. 
Now, we impose force balance at the perturbed plasma-vacuum interface, finding:

$$
\begin{aligned}
& \frac{\left(\rho \omega^{2}-F^{2}\right)\left[\rho \omega^{2}\left(\gamma p+B^{2}\right)-\gamma p F^{2}\right]}{\Delta} \frac{\left(\mathbf{r} \xi_{r}\right)^{-}}{\left(\mathbf{r} \xi_{\mathbf{r}}\right)} \mid \mathbf{r}=\mathbf{r}_{\mathbf{p}}=\frac{\mathrm{B}_{\theta}^{v^{2}}+\mathrm{B}_{\theta}^{2}}{\mathbf{r}_{\mathbf{p}}} \\
& +\left(k B_{z}^{v}+\frac{m}{r_{p}} B_{\theta}^{v}\right)^{2} \frac{\left[K_{m}\left(|k| r_{p}\right)-I_{m}\left(|k| r_{p}\right) \frac{K_{m}^{\prime}\left(|k| r_{w}\right)}{I_{m}^{\prime}\left(|k| r_{w}\right)}\right]}{\left[K_{m}^{\prime}\left(|k| r_{p}\right)-I_{m}^{\prime}\left(|k| r_{p}\right) \frac{K_{m}^{\prime}\left(|k| r_{w}\right)}{I_{m}^{\prime}\left(|k| r_{w}\right)}\right]} \\
& +\left[\frac{2 k B_{\theta}\left(k B_{\theta}-\frac{m}{r} B_{z}\right) \rho \omega^{2} B^{2}}{r \Delta}+\frac{2 k{ }^{2} p B_{\theta}\left(k B_{\theta}-\frac{m}{r} B_{z}\right)\left(\rho \omega^{2}-F^{2}\right)}{r \Delta}\right]_{r}=r_{p},
\end{aligned}
$$

where $p, p, B_{\theta}$ and $B_{z}$ are equilibrium quantities in the plasma region.

For the P-V-W system, Eq. (6), the regularity condition at $r=0$,

$$
\xi_{r}(r=0)=\text { finite, }
$$

and Eq. (17) determine $w$ and $E_{T}(r)$ for given $m, k$, and equilibrium profiles. Equations (14) - (16) determine the perturbed vacuum magnetic field; Eq. determines the position and shape of the perturbed interface.

\section{NUMERICAL RESULTS}

In this esction we present numerical results obtained from the dispersion differential equation for the P-W system, Eqs. (6), (10), and (11), and the P-V-W system, Eqs. (6), (17), and (18).

In $\mathrm{Fig}$. 1, the growth rate squared is plotted against axial wavevector, $\mathrm{ka}$, for $m=1, \beta=0.5, \mu=0.3, r_{p}=2.0 r_{0}$, and $r_{w}=4.0 r_{0}$. For $|\mathrm{ka}| \geq$ 0.06 , the elgenvalues for the $P-W$ and $P-V-W$ system do not differ preceptively 
and only one solution, $\omega(k)$, lis showr; for these modes the singular surface, defined by $\left.F\left(r_{s}\right) \equiv\left(k B_{z}+\frac{m}{r} B_{\theta}\right)\right|_{r_{s}}=0$, occurs at $r=r_{s}<r_{p}$. The growth rates for the $P-W$ and $P-V-W$ systems differ considerably when $|k a|<0.06$; in this case the singular surface occurs at $r=r_{s}>r_{p}$. The singular surface lies in the vacuum region of the $P-V-W$ system and the growth rates of these modes are considerably larger than those of the P-W system. Both the P-W and $\mathrm{P}-\mathrm{V}-\mathrm{W}$ system are stable when the singular surface reaches the conducting wall $r_{s}=r_{w}(k a=-0.014)$. Thus, the two configurations are unstable for the same range of $k a$, but the growth rates of the unstable modes can be considerably different for the two systems.

Figure 2 shows the elgenfunctions, $\xi_{r}(r)$, of the unstable modes of the $P-W$ and $P-V-W$ systems when $k a=-0.07, m=1, B=0.5, \mu=0.3, r_{p}=2.0 r_{0}$, and $r_{w}=4.0 r_{0}$. In this case the eigenvalues are almost identical, and the eigenfunctions do not differ perceptibly for $r / r_{0}<2.0$. For the P-V-W system, the plasma solution is joined to the vacuum solution at $r / r_{0}=2.0$; the displacement $\xi$ is not defined for $r / r_{0}>2.0$. In the $P-W$ system, the displacement is defined for the entire domain $0<r / r_{0}<4.0$. The singular surface for these modes lies in the plasma region, $r_{s}<r_{p}$.

The flow flelds associated with the unstable modes of the $P-W$ and $P-V-W$ systems are shown in Figs. 3 and 4, respectively, for the parameter value given in Fig. 2. The region $r / r_{0}<2.0$ is shown. An arrow depicts the displacement vector associated with the position at the tail of the arrow. The two flow patterns do not differ perceptively. These flow flelds are Indicative of internal or bulk modes which translate the cross section of the plasma; recirculation occurs in the vicinity of the singular surface, $r / r_{0}-$ 2.0 .

Figure 5 shows the elgenfunctions, $\xi_{r}(r)$, of the unstable modes of the $P-V-W$ and $P-W$ systems when the singular surface occurs in the vacuum region, $r_{s}>r_{p} ; \mathrm{ka}=-0.04, \mathrm{~m}=1, \beta=0.5, \mu=0.3, r_{p}=2.0 r_{0}$, and $r_{w}=4.0 r_{0}$ In this case, the eigenvalues of the two configurations differ considerably. The elgenfunctions reflect the differences in the elgenvalues and are considerably different also. In the P-W system, the eigenfunction resembles the one shown in Fig. 2; but in the P-V-W system, the elgenfunction now attains its maximum value at the edge of the plasma, whereas in Fig. 2 , it attains its minimum value there. A perturbation which decreases :apidly with distance from the boundary is characteristic of a surface mode.2,3 


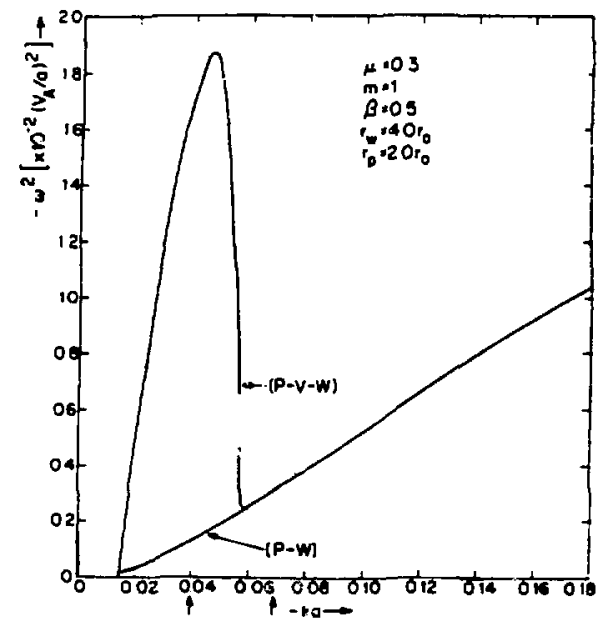

Fig. 1.

Growth rate squared versus axial wavevector. The two curves do not differ perceptively when $|\mathrm{ka}|>0.06$. Mode structures are examined in Figs. 2-4 for $k a=-0.07$, and in F1gs. $5-7$ for $k a=-0.04$.

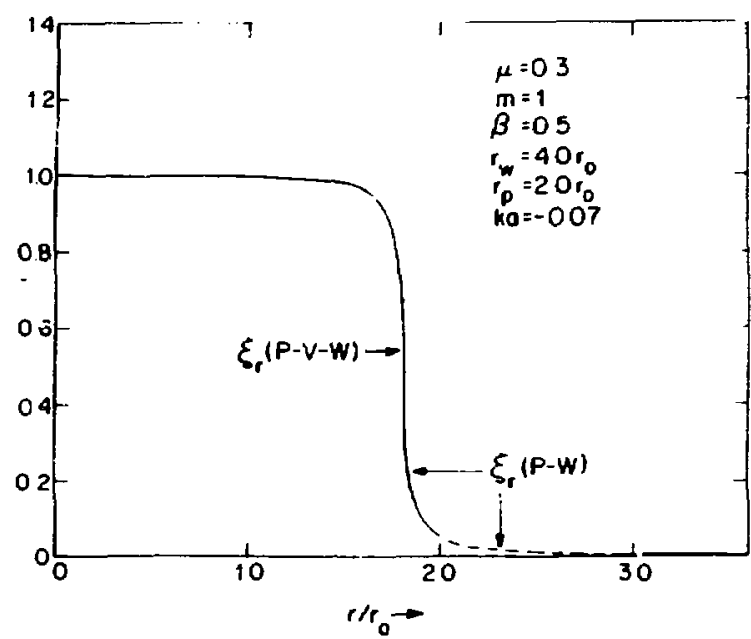

Fig. 2 .

Elgenfunctions assoclated with unstable modes with ka $=-0.07$. The two functions do not differ perceptively in the range $0<r / r_{0}<2.0$.
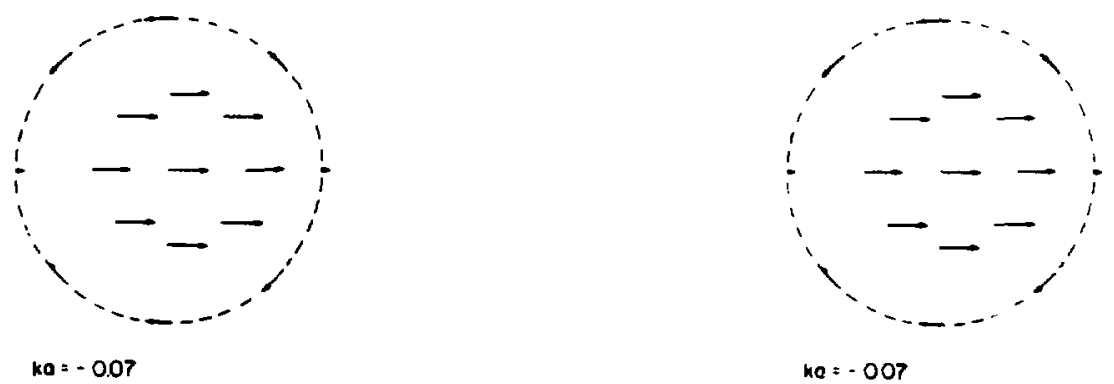

Fig. 3.

Flow pattern for the $\mathrm{P}-\mathrm{W}$ mode. The region $r / r_{0}<2.0$ is shown.

F1g. 4.

Flow pattern for the $P-V-W$ mode. The region $r / r_{0}$ ? 2.0 is shown. 
Other features of the unstable modes may be distingulshed from their flow flelds which are shown in Fig. 6, for the P-H system, and Fig. 7, for the P-V-W system; parameter values are the same as in Fig. 5 . The region $r / r_{0}<$ 2.0 is shown. For the P-W system, the flow field resembles the one in Fig. 3. Th: flow causes transiation of the cross section of the plasma; recirculation occurs near the singular surface, $r \simeq r_{s}>r_{p}$, which 18 outside the field of vlew. Th1s 18 an internal or bulk mode. The flow pattern of the unstable

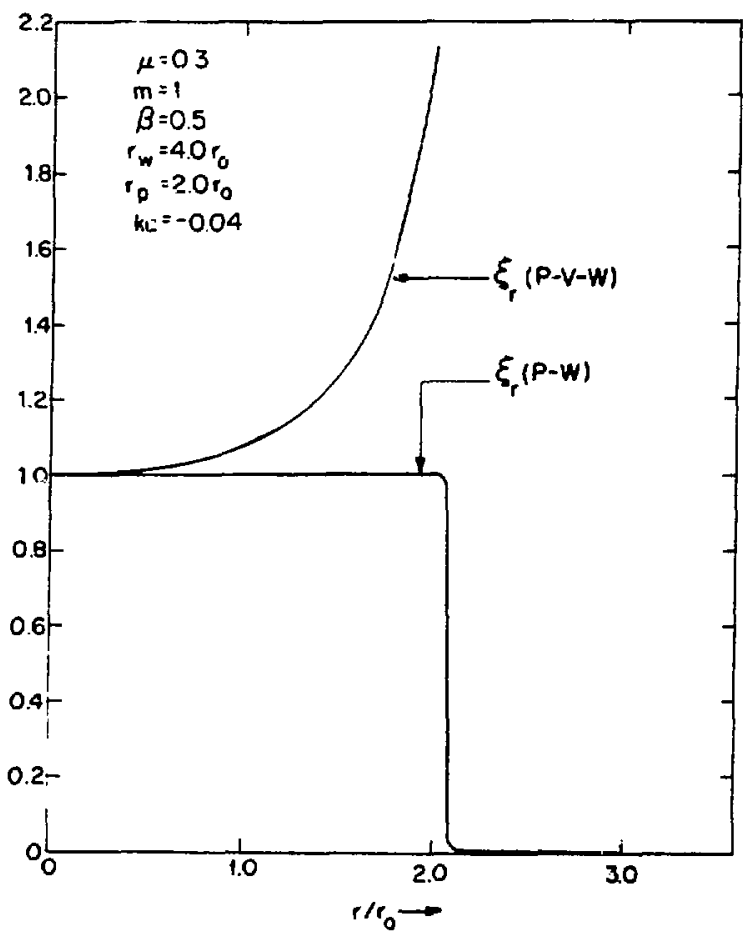

Fig. 5. Eigenfunctions associated with unstable modes with ka $=-0.04$. The $P-V-W$ elgenfunction is typical of a surface mode.

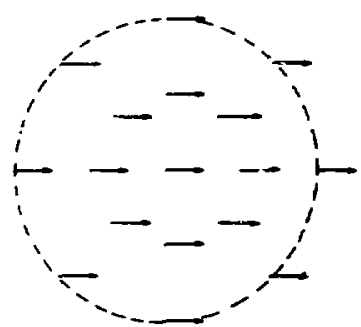

$\mathrm{kg}=-0.04$

F1g. 6 .

Flow pattern for the $P-N$ mode. The region $r / r_{0}<2.0$ is shown.

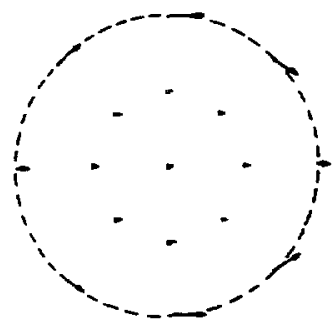

$k a=-0.04$

F1g. 7.

Flow pattern for the $\mathrm{P}-\mathrm{V}-\mathrm{W}$ mode. The region $r / r_{0}<2.018$ shown. 
P-V-W mode, Fig. 7, is completely different. There is no recirculation; rather, the mode peels-off the edge of the plasma column.

Figures 5 and 7 indicate that the $P-V-W$ system supports unstable surface modes. The growth rates of the modes depend upon the properties of plasma in the vicinity of the interface; this is a general feature of surface modes.

\section{DISCUSSION}

We have compared the stability properties of two screw pinch equilibria In order to ascertain the influence of a vacuum region on MHD stability. We have found that the stability boundary for this pinch 18 not affected when the outer tenuous plasma is replaced by a vacuum region. The presence of a vacuum region does affect the nature of the unstable modes and their growth rates, however. The $\mathrm{P}-\mathrm{W}$ system supports only bulk modes; but the P-V-W system supports both tulk modes and surface modes. Surface modes have no counterpart In the P-W system. On the other hand, some of the bulk modes of the P-W system nave no counterpart in the $P-V-W$ system. Some of the $P-W$ bulk modes are unstable, but they cannot exist in the P-V-W configuration; unstable surface modes appear in the latter configuration which cannot exist in the former. Thus, the presence of a vacuum region does not affect the stability boundary of the pinch, but 1 t does affect the nature of the unstabis modes and their growth rates.

\section{ACKNOWLEDGMENTS}

The author thanks D. Barnes, G. Miller and M. Schmidt for useful discussions.

\section{REFERENCES}

1. J. P. Freidberg, Phys. Fluids 13, 1812 (1970).

2. W. P. All1s, S. J. Buchsbaum, A. Bers, Waves In Antsotropic Plasmas (M.I.T. Press, Cambridge, MA, 1963), p. 214-222.

3. A. W. Trivelpiece, Slow-Wave Propagation in Plasma Waveguldes (San Francisco Press, San Francisco, CA, 1967), Chap. 4. 